\section{Ultrasonography of the lower extremity veins: anatomy and basic approach}

\author{
Dong-Kyu Lee, Kyung-Sik Ahn, Chang Ho Kang, Sung Bum Cho \\ Department of Radiology, Korea University Anam Hospital, Korea University College of \\ Medicine, Seoul, Korea
}

Ultrasonography is an imaging modality widely used to evaluate venous diseases of the lower extremities. It is important to understand the normal venous anatomy of the lower extremities, which has deep, superficial, and perforating venous components, in order to determine the pathophysiology of venous disease. This review provides a basic description of the anatomy of the lower extremity veins and useful techniques for approaching each vein via ultrasonography.

Keywords: Anatomy; Lower extremity; Ultrasonography; Vein

\section{Introduction}

Ultrasonography (US) is the most useful and widely used modality for evaluating venous diseases of the lower extremities [1-3]. The examiner should be familiar with the normal venous anatomy of the lower extremities, which is somewhat different from the corresponding arterial anatomy. An understanding of the lower extremity venous system is important for diagnosing and determining the pathophysiology of venous disease.

The venous system of the lower extremities is classified into three groups according to the relationship to the muscular fascia that surrounds the calf and leg muscles [4-6]. The veins that lie beneath the muscular fascia and drain the lower extremity muscles are the deep veins, those that are above the muscular fascia and drain the cutaneous microcirculation are the superficial veins, and those that penetrate the muscular fascia and connect the superficial and deep veins are the perforating veins (Fig. 1). These groups are closely related to one another. This article reviews the anatomy of the deep, superficial, and perforating veins in the lower extremities for US and provides practical tips for approaching each vein to evaluate venous disease of the lower extremities.

\section{General Considerations for the US Examination of Veins}

In contrast to arteries, veins have a weaker muscular layer with less elastic walls, and therefore completely collapse when the vein is compressed by the transducer (Fig. 2A). The compressibility of veins and pulsation of the arteries can be a way to discriminate them in US. In addition, veins have valves that play an important role in preventing reflux of the venous flow (Fig. 2B). The normal flow is directed from distal to proximal and from superficial to deep. Usually, vascular flow is not seen on grayscale US, and the venous lumen presents as anechoic. However, venous flow sometimes presents as echogenic, which can reflect red blood cell aggregation $[7,8]$ and should not be confused with

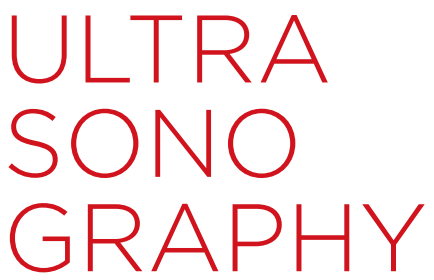

\section{REVIEW ARTICLE}

https://doi.org/10.14366/usg. 17001 pISSN: 2288-5919 e elSSN: 2288-5943 Ultrasonography 2017;36:120-130

Received: January 5, 2017

Revised: January 29, 2017

Accepted: January 30, 2017

Correspondence to: Kyung-Sik Ahn, MD, Department of Radiology, Korea University Anam Hospital, Korea University College of Medicine, 73 Inchon-ro, Seongbuk-gu, Seoul 02841, Korea

Tel. +82-2-920-6540

Fax. +82-2-929-3796

E-mail: glassesik@gmail.com

This is an Open Access article distributed under the terms of the Creative Commons Attribution NonCommercial License (http://creativecommons.org/ licenses/by-nc/3.0/) which permits unrestricted noncommercial use, distribution, and reproduction in any medium, provided the original work is properly cited.

Copyright (C) 2017 Korean Society of Ultrasound in Medicine (KSUM)

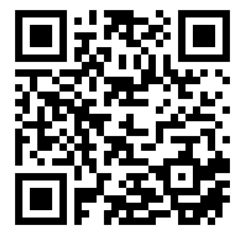

How to cite this article: Lee DK, Ahn KS, Kang CH, Cho SB. Ultrasonography of the lower extremity veins: anatomy and basic approach. Ultrasonography. 2017 Apr;36(2):120-130. 

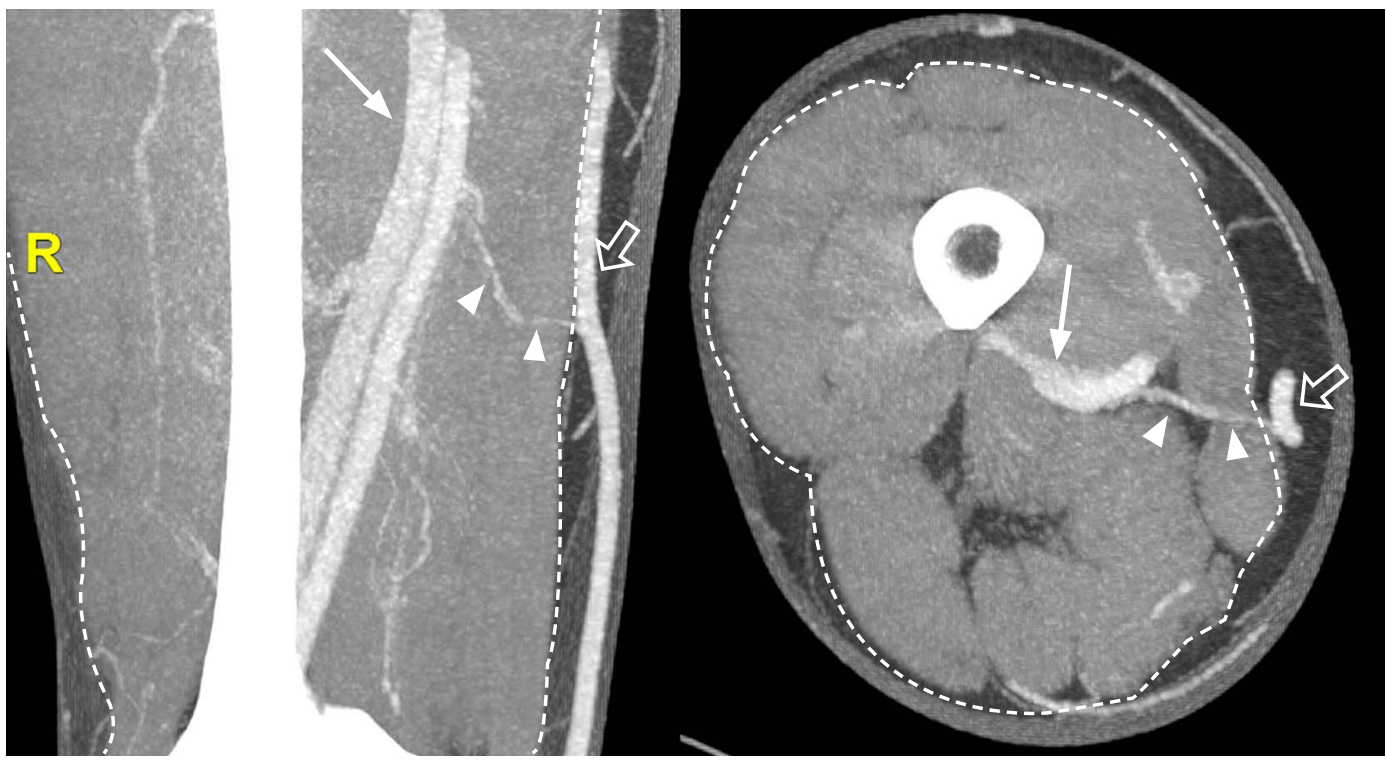

Fig. 1. Normal computed tomography venography of the lower extremities. The venous system of the lower extremities is classified into three groups: deep veins, which lie beneath the muscular fascia (dotted lines); superficial veins, which are situated above the muscular fascia; and perforating veins, which penetrate the muscular fascia and connect the superficial and deep veins. Note the deep vein (femoral vein, arrows), superficial vein (great saphenous vein, open arrows), and perforating vein (femoral canal perforator, arrowheads) of the thigh.

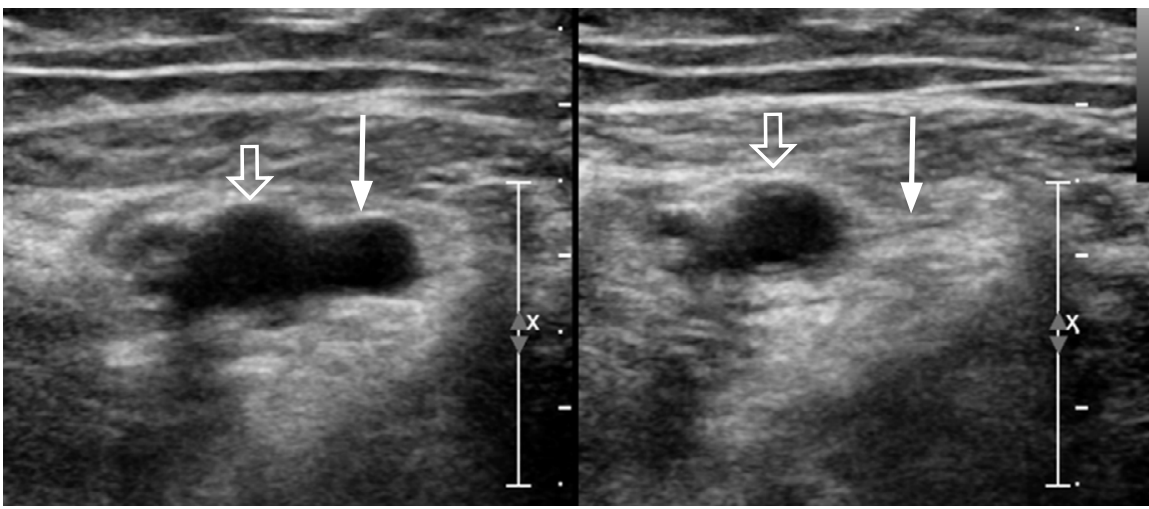

A

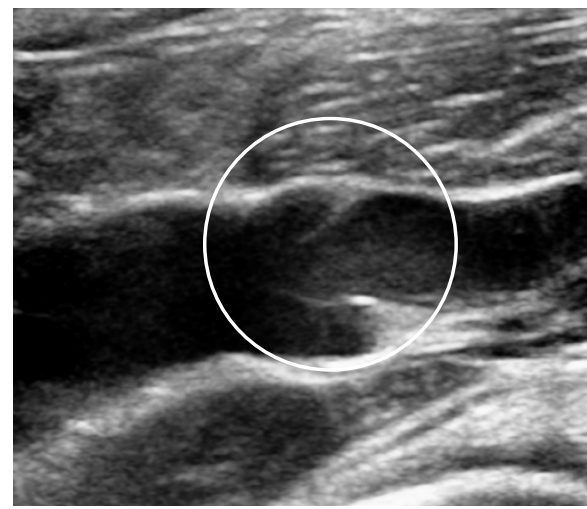

B

Fig. 2. Ultrasonographic findings of normal veins.

A. In contrast to arteries (open arrows), veins (arrows) have a weaker muscular layer with less elastic walls, and therefore completely collapse when compressed by the transducer. B. Veins contain valves that play an important role in preventing venous reflux.

thrombosis. This phenomenon is found especially frequently in conditions of slow venous flow. Continuous observation without transducer movement or identifying the compressibility of the lumen can be helpful to differentiate this condition from true thrombosis.

The selection of a transducer for evaluating the lower extremity veins is a trade-off between resolution and beam penetration. Generally, a frequency of $5 \mathrm{MHz}$ or greater is recommended, but sometimes a lower-frequency transducer is required for deeppenetration views in obese, edematous, or muscular patients [1].

\section{Deep Veins}

\section{Anatomy}

The major deep veins of the lower extremities follow the course of the corresponding arteries. The deep venous system of the calf includes the anterior tibial, posterior tibial, and peroneal veins. In the calf, these deep veins present as pairs on both sides of the artery. The posterior tibial vein receives blood from the medial and lateral plantar vein and drains the posterior compartment of the leg and plantar surface of the foot. This vein lies behind the tibia and 
joins the popliteal vein at the posterior knee. The anterior tibial vein is the upward continuation of the dorsal pedal vein. It runs along the anterior compartment of the leg just above the interosseous membrane between the tibia and the fibula, and joins the posterior tibial vein to form the tibioperoneal trunk and popliteal vein. The peroneal vein runs along the posteromedial aspect of the fibula and joins the posterior tibial vein.

The popliteal vein is formed by the junction of the anterior and posterior tibial veins at the lower aspect of the posterior knee. It ascends along the posterior aspect of the knee and the distal aspect of the anteromedial thigh. The popliteal vein is located medial to the artery in the lower knee, superficial to the artery at the posterior knee, and to the lateral side above the knee.

After the popliteal vein goes into the adductor hiatus, it is referred to as the femoral vein. The term superficial femoral vein is no longer recommended, because this vein is not a superficial vein, but rather a deep vein [9-11]. In the lower part, it lies lateral to the artery; in the mid-part, behind the artery; and in the upper part, medial to the artery. The deep femoral vein from the inner thigh, running along the deep femoral artery, joins the femoral vein and forms the common femoral vein, which is located medial to the common femoral artery. The inguinal ligament is the landmark that divides the common femoral vein from the external iliac vein (Fig. 3).

\section{Approach}

It is convenient for the US examination to begin with the patient in a supine or semi-Fowler position. The reverse Trendelenburg position, if possible, is also recommended, as it facilitates venous filling in the lower extremities and makes the veins dilate. External rotation of the hip and slight flexion of the knee helps to decrease muscle tension and is good both for exposing the deep veins in the medial thigh, posterior knee, and calf, and for the compression maneuver (Fig. 4A).

Veins drain blood in the distal-to-proximal direction to the right side of the heart. However, it is convenient to examine the deep veins from a proximal to distal direction because the proximal veins are larger in diameter and easy to detect. At the level of the inguinal ligament, in the transverse view, the examiner can see the common femoral vein on the medial side of the artery (Fig. 4B). Information about the flow pattern of the vein can be assessed in the longitudinal view with Doppler US [12]. Following the common femoral vein, it bifurcates into the deep femoral vein and the femoral vein (Fig. 4C). In the more distal part of the medial thigh, only the femoral vein is visible (Fig. 4D).

When the transducer approaches the popliteal fossa of the posterior knee, the popliteal vein is visible. The popliteal vein is superficial to the popliteal artery at the posterior knee (Fig. 4E).

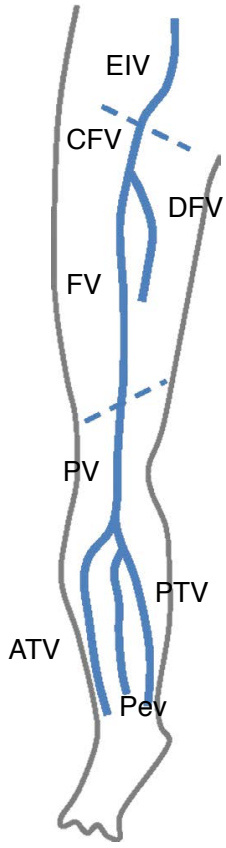

Fig. 3. Schematic image of lower extremity deep veins. Although lower extremity veins form a continuous structure, they are named individually according to their closest anatomic landmark. The inguinal ligament (upper dotted line) is the anatomic landmark between the external iliac and common femoral veins, and the adductor hiatus (lower dotted line) is the anatomic landmark between the femoral and popliteal veins. EIV, external iliac vein; CFV, common femoral vein; $F V$, femoral vein; DFV, deep femoral vein; $P V_{\text {, }}$ popliteal vein; ATV, anterior tibial vein; PTV, posterior tibial vein; PeV, peroneal vein.

Duplicated femoral veins or popliteal veins can be seen [13]. Because a duplicated venous segment may result in false-negative results, both lumens should be carefully examined (Supplementary Fig. 1). Manual vein compression is recommended every 3-4 cm [3].

When tracing the popliteal vein downward, two posteriorly branching veins are found in the calf from the posteromedial approach. The vein along the tibia is the posterior tibial vein, and the vein along the posteromedial aspect of the fibula is the peroneal vein. In a US approach, the cortical shadow of the tibia and fibula can be used as a bony landmark. The paired veins are present on both sides of the artery (Fig. 4F). After stretching the patient's leg, the anterior tibial vein can be visualized from an anterolateral approach (Fig. 5). Just above the echogenic interosseous membrane between the tibia and the fibula, the anterior tibial artery and vein can be found. If the posterior tibial vein cannot be traced in the proximal to distal direction, it is helpful to trace the vein upward from the posterior to the medial malleolus, where this vein is located more superficially. If the popliteal and calf veins are not visualized well in the supine-based position, the prone or decubitus position 


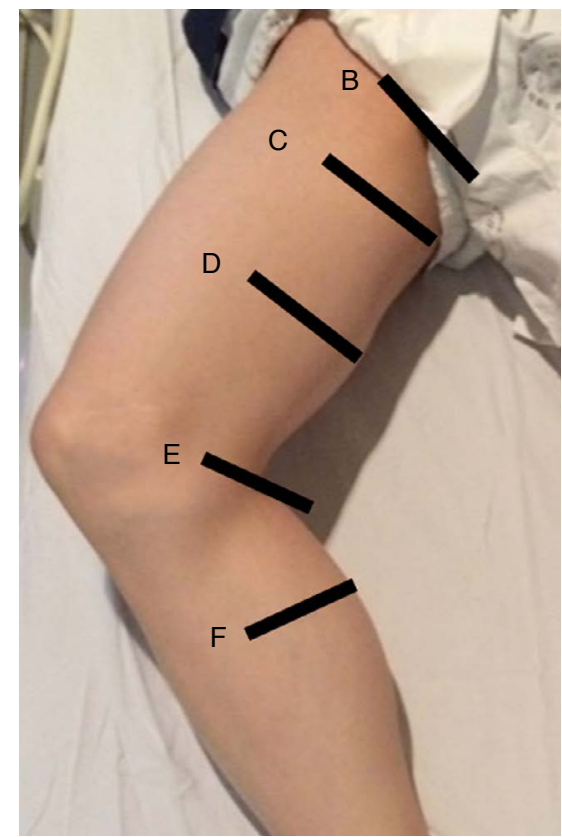

A

Fig. 4. Demonstration of side-by-side transverse ultrasonography (noncompression and compression views) and the corresponding sonic window in computed tomography venography based on transducer placement.

A. Patient position and schematic representation of the transducer locations are shown here $(B, C, D, E$, and F). B. The common femoral vein is seen at the level of the inguinal ligament on the medial side of the common femoral artery, which is round and pulsatile. The vein collapses upon compression (arrows). C. Upon following the common femoral vein downward, it bifurcates into the deep femoral vein (open arrows) and the femoral vein (arrows).
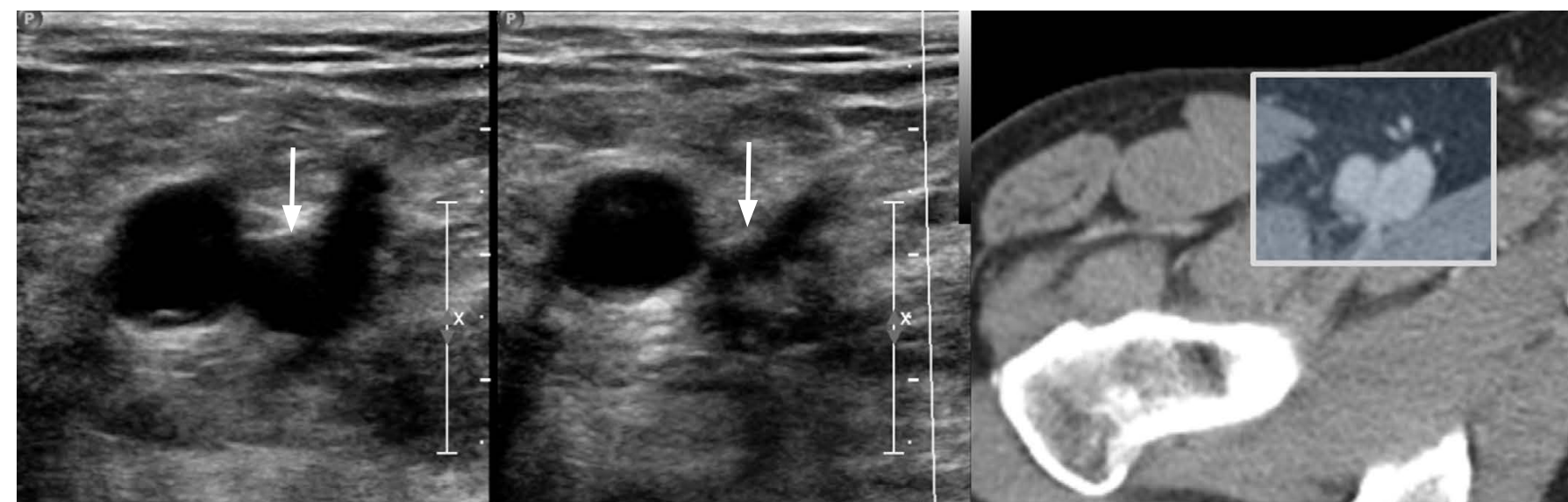

B
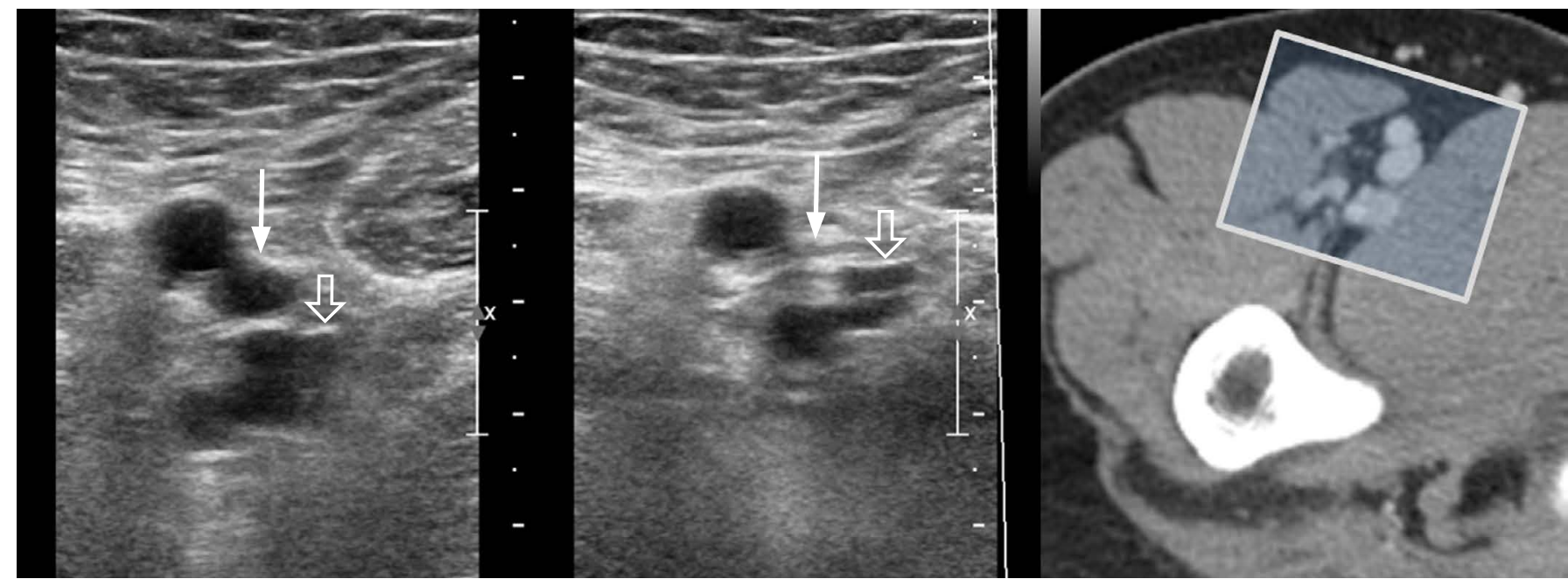

C 


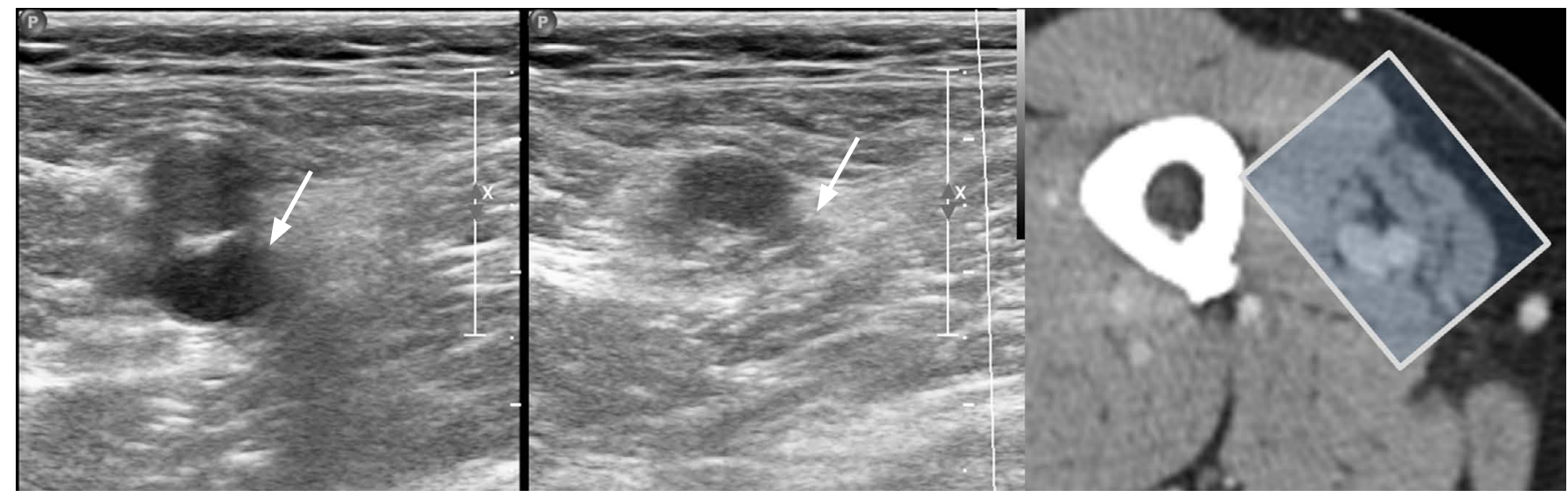

D

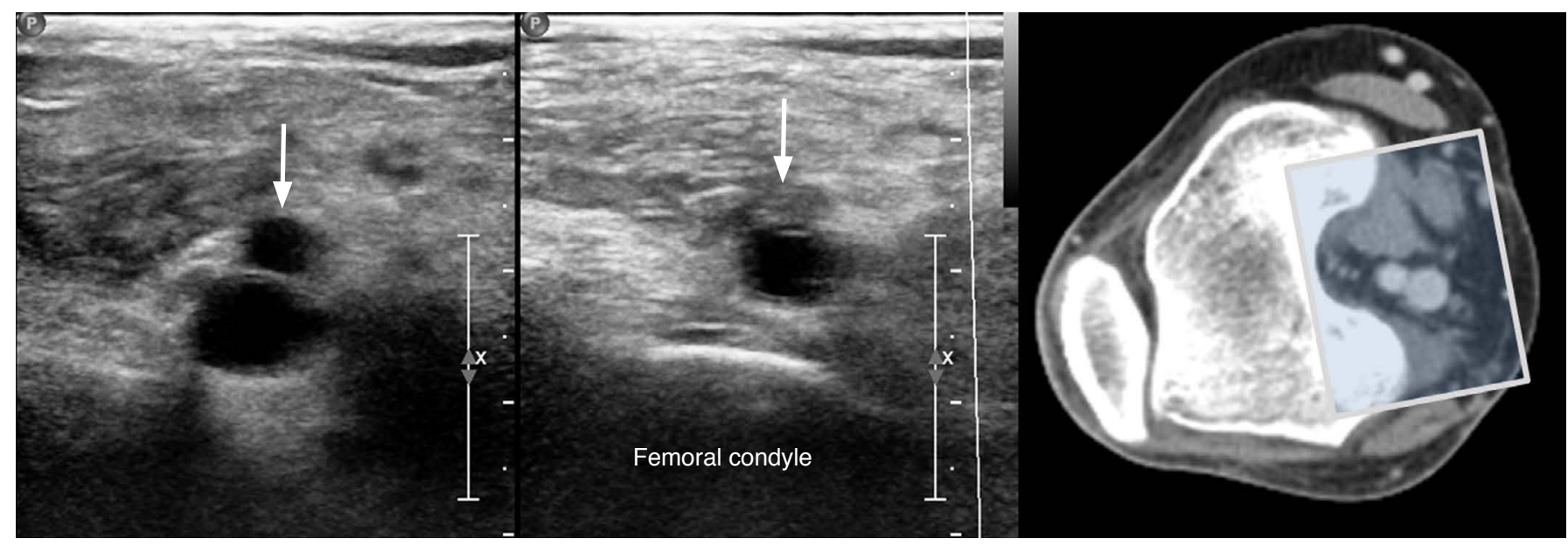

E
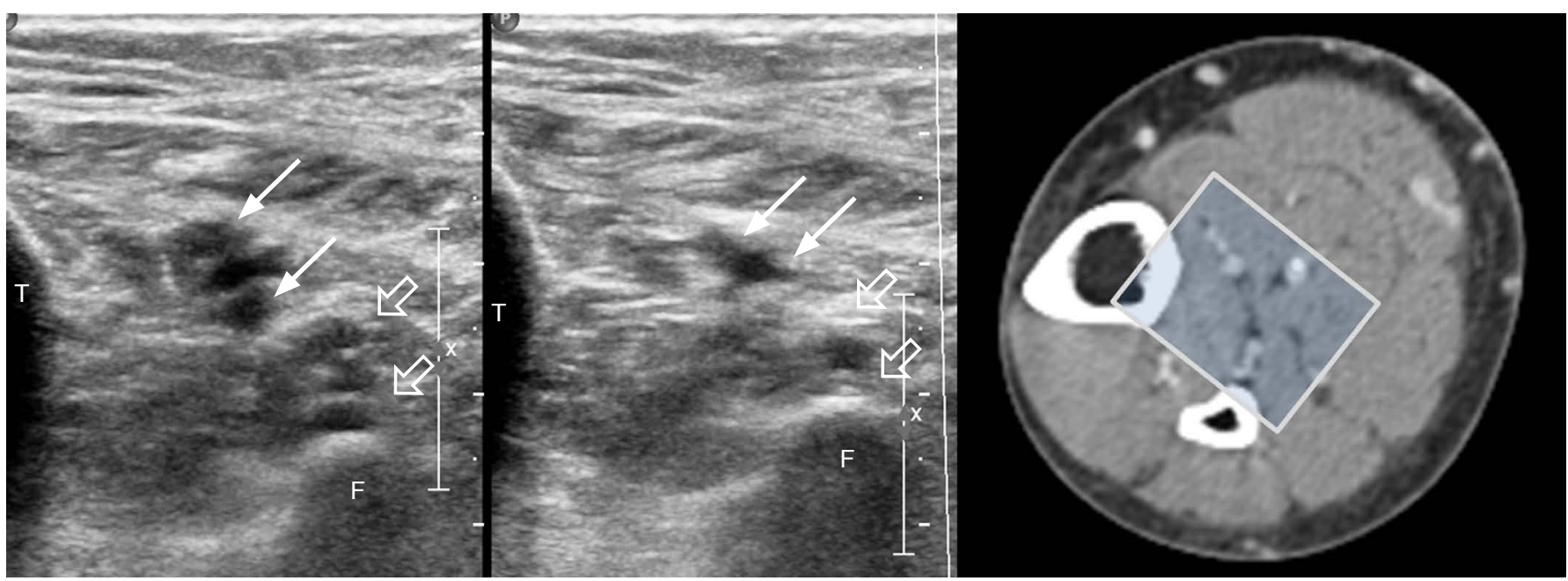

$\mathrm{F}$

D. Only the femoral vein (arrows) is visible in the more distal part of the medial thigh. E. When the transducer approaches the popliteal fossa in the posterior knee, the popliteal vein (arrows) becomes visible. F. Tracing the popliteal vein downward from the posteromedial approach shows two posteriorly branching veins in the calf. The vein along the tibia $(T)$ is the posterior tibial vein (arrows) and the vein along the fibula $(\mathrm{F})$ is the peroneal vein (open arrows). The veins are present on both sides of the artery. 


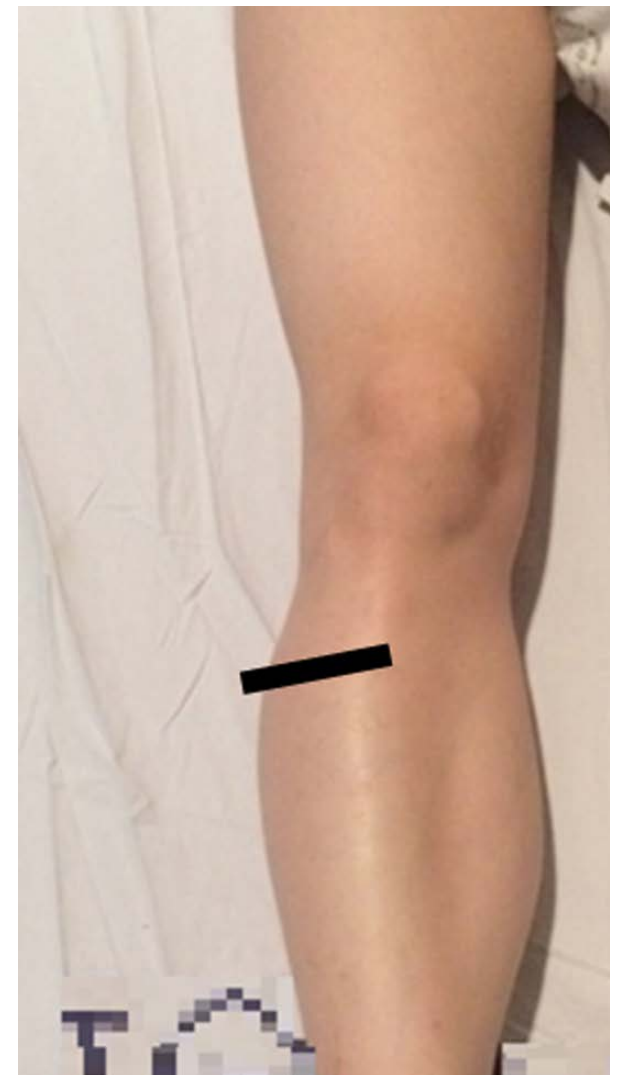

A
Fig. 5. Ultrasonographic findings of the anterior tibial vein.

A. Stretching of the patient's leg permits approach to the anterior tibial vein from the anterior side. B. Above the interosseous membrane (arrowheads) between the tibia (T) and the fibula (F), the anterior tibial vein (arrows) and artery (open arrows) are visible. The sonic window is demonstrated through computed tomography venography.

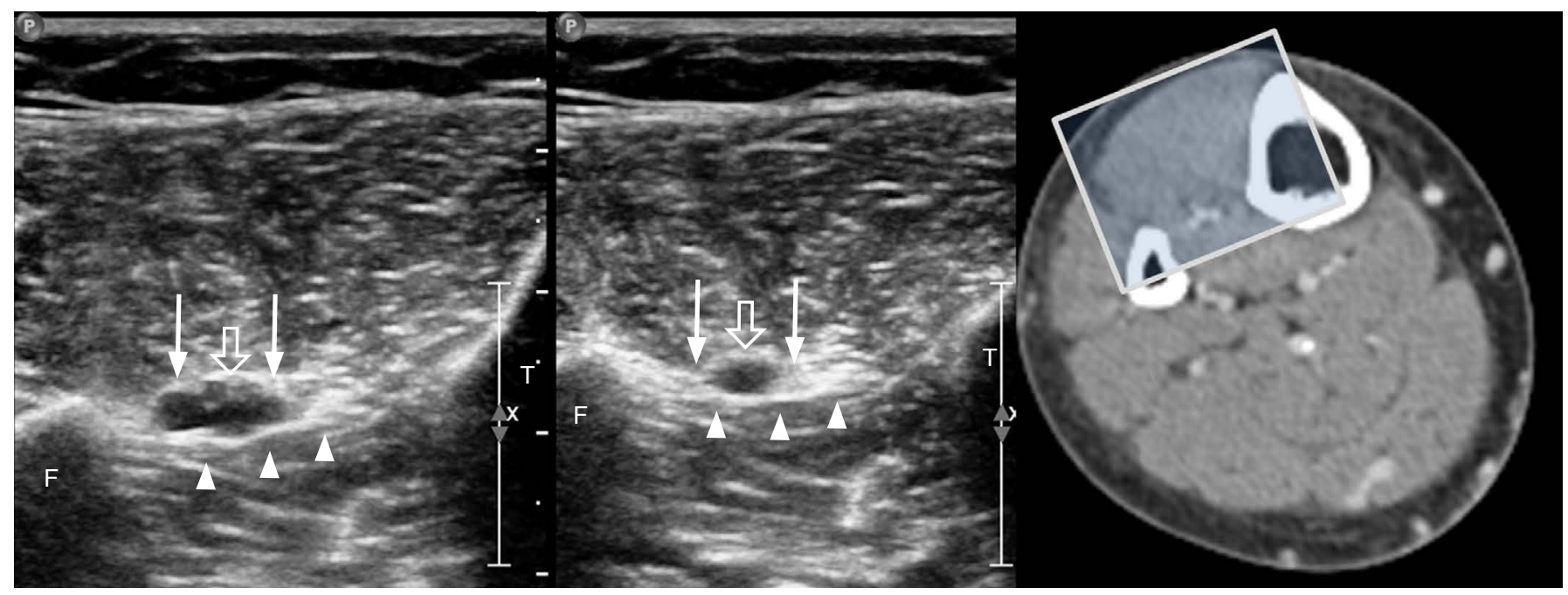

B

can also be helpful. The distal femoral vein at the level of the adductor hiatus can be difficult to evaluate because of its depth and the transducer location.

\section{Superficial Veins}

\section{Anatomy}

The two major superficial veins of the lower extremities are the great saphenous vein (GSV) and small saphenous vein (SSV). The terms "greater saphenous vein," "long saphenous vein," "lesser 


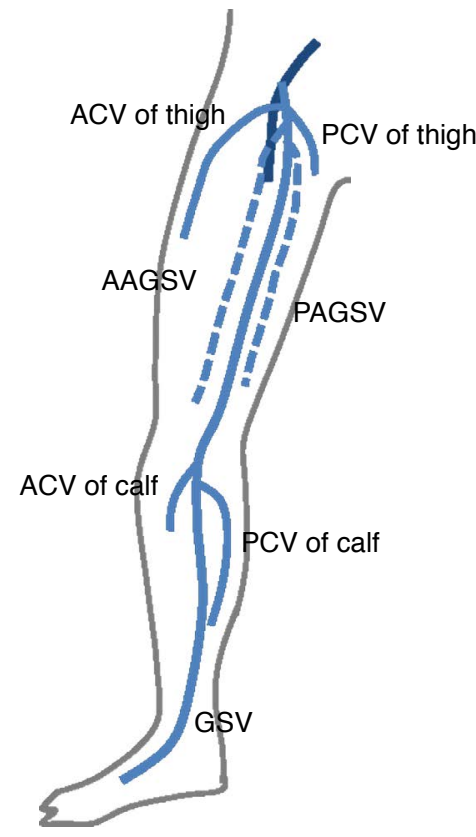

A

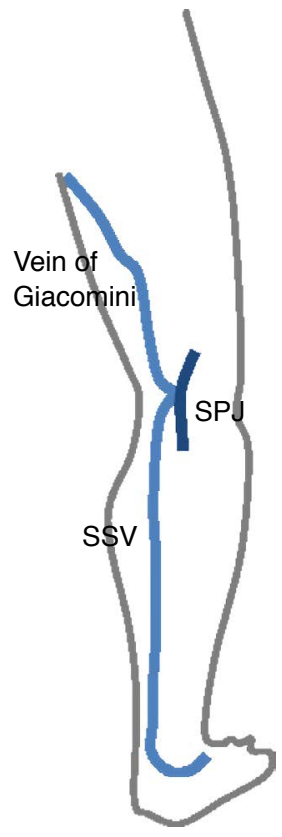

B
Fig. 6. Schematic images of the superficial veins of the lower extremities.

A. The great saphenous vein (GSV) arises from the medial aspect of the dorsal pedal venous arch, ascends anteriorly to the medial malleolus, passes posteromedially to the knee, and ascends medially in the thigh to join the common femoral vein through the saphenofemoral junction. Tributaries of the GSV are named according to their course. A tributary parallel to the GSV is called an accessory GSV. A tributary that courses obliquely is called a circumflex vein. ACV, anterior circumflex vein; $P C V$, posterior circumflex vein; AAGSV, anterior accessory great saphenous vein; PAGSV, posterior accessory great saphenous vein. B. The small saphenous vein (SSV) arises from the dorsal pedal arch and ascends along the middle of the calf and ends in the popliteal vein through the saphenopopliteal junction (SPJ). Before it penetrates the muscular fascia, the SSV may branch out a cranial extension, known as the vein of Giacomini, which goes upward to join the GSV. saphenous vein," and "short saphenous vein" are no longer recommended for the purpose of standardizing terminology $[10,11]$.

The GSV begins in the medial marginal vein of the dorsum of the foot, ascends anteriorly to the medial malleolus, and passes posteromedially to the knee. The vein then ascends medially in the thigh to perforate the muscular fascia and join the common femoral vein at the saphenofemoral junction, a few centimeters distal to the inguinal ligament. Tributaries of the GSV are variable between individuals, but there are general rules for naming them [10]. If the course of the tributary is parallel to the GSV, it is called an accessory GSV (an anterior accessory GSV or a posterior accessory GSV). When the tributary courses obliquely, it is called the circumflex vein (the posterior thigh circumflex vein or the anterior calf circumflex vein) (Fig. 6A). However, despite attempts to standardize the terminology, various terms have been used and many of the tributaries are difficult to name.

The SSV arises from the dorsal pedal arch and ascends posterolaterally from behind the lateral malleolus. It ascends along the middle of the calf and ends in the popliteal vein in the posterior knee. However, there are many variations in the termination of the SSV, including without connection to the popliteal vein $[5,14]$. Before it penetrates the muscular fascia, it may branch out a cranial extension that goes upward to join the GSV through the posterior thigh circumflex vein (the vein of Giacomini) (Fig. 6B).

\section{Approach}

Insufficiency of the superficial veins should be evaluated with the subject in the erect position, as the supine position may underestimate or miss the reflux of the venous flow (Fig. 7A). The reverse Trendelenburg position can be used if an erect position is impossible. The examined leg should be in a non-weightbearing position [1]. The saphenofemoral junction is visible on the anteromedial surface of the common femoral vein in the transverse view. A longitudinal view of the saphenofemoral junction (Fig. 7B) is useful for assessing reflux of the terminal valve with the help of Doppler US (Supplementary Fig. 2). Normally, the terminal valve at the saphenofemoral junction prevents backward flow into the GSV. After a provocation test, such as the Valsalva maneuver, retrograde flow in the proximal GSV persisting for more than 0.5 seconds is defined as pathologic reflux $[4,15]$.

There is an echogenic fascia surrounding the GSV that is bordered deeply by the muscular fascia and superficially by the saphenous fascia. This saphenous compartment is readily visualized on US and is described as having an "Egyptian eye" appearance (Fig. 7C). The saphenous compartment is also visible at the knee level, above the tibia and medial gastrocnemius (Fig. 7D). Unfortunately, however, there are many variations in the relationship of the fascial compartment to the GSV. Sometimes there is a large tributary outside of the saphenous compartment, while the distal GSV is hypoplastic or even absent $[14,16]$.

The SSV is visible at the middle of the gastrocnemius belly in 


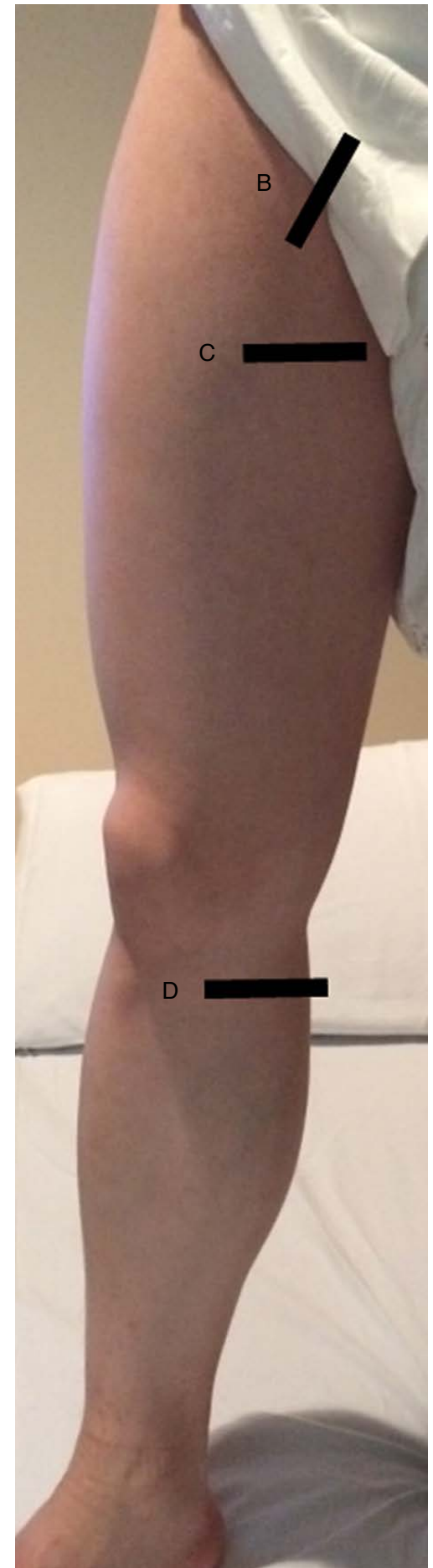

A
Fig. 7. Ultrasonographic findings of the great saphenous vein (GSV).

A. Patient position (standing) and schematic representation of the transducer locations are shown here. B. Longitudinal view of the saphenofemoral junction and the corresponding sonic window in computed tomography venography based on transducer location are demonstrated. In most cases, a terminal valve (arrows) near the saphenofemoral junction prevents backward flow into the great saphenous vein. CFV, common femoral vein. C. Examination of the GSV shows the echogenic fascia surrounding the GSV, which is bordered deeply by the muscular fascia (arrows) and superficially by the saphenous fascia (arrowheads). This saphenous compartment is readily visualized on ultrasonography and is described as having an "Egyptian eye" appearance. D. Transverse ultrasonography examination at the level of the knee shows the saphenous compartment (arrowheads) overlying the tibia ( $T$ ) and the muscle fascia of the medial gastrocnemius $(G)$.

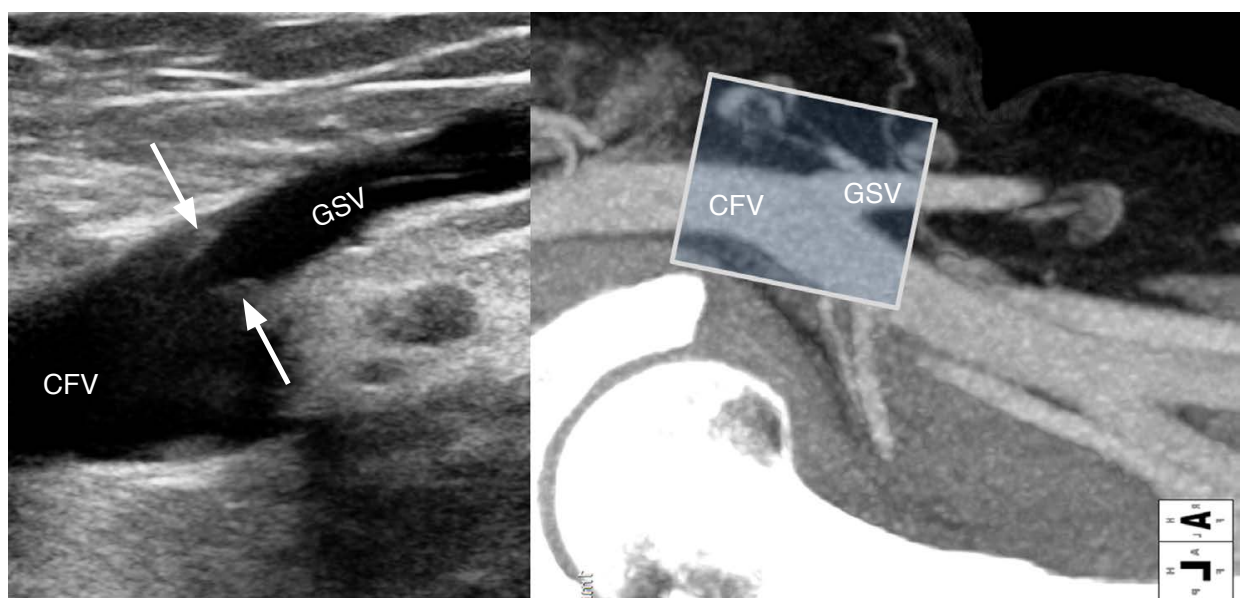

B
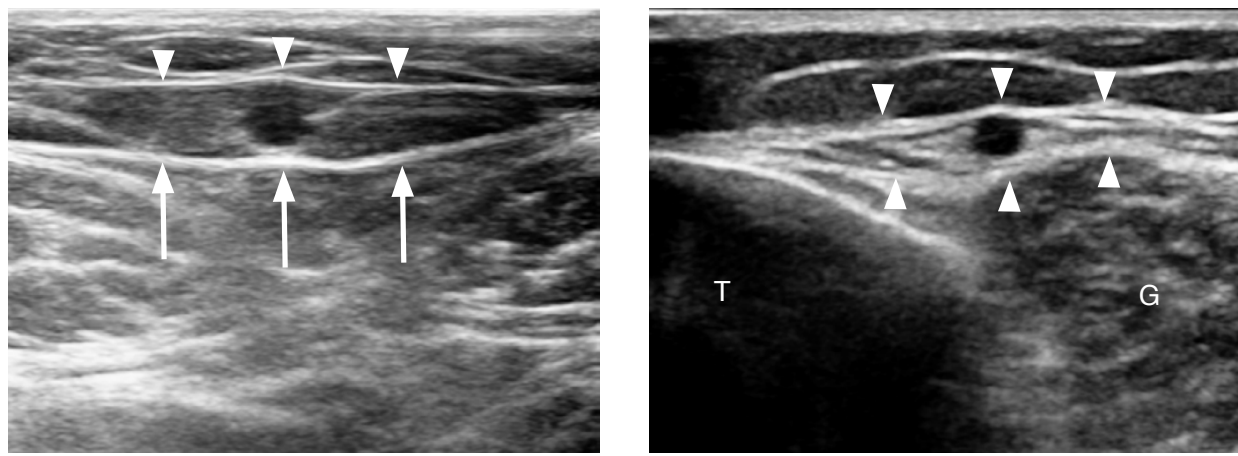

C the fascial trunk (Fig. 8). In tracing the SSV upward, it enters into the popliteal vein. The longitudinal view of the saphenopopliteal junction, like the saphenofemoral junction of the GSV, is useful for assessing insufficiency of the SSV. In the saphenopopliteal junction, calf squeezing augments the venous flow at first, followed by retrograde flow. As described above, however, there are many anatomical variations in this region, and the saphenopopliteal junction may be absent or rudimentary $[5,14]$. 


\section{Perforating Veins}

\section{Anatomy}

The perforating veins connect the deep veins with the superficial veins and direct the flow from the superficial to the deep system. There are numerous perforators in the leg. According to a consensus statement [10], perforators are named after their locations. Major groups classify perforators according to their longitudinal location as ankle, leg, knee, and thigh perforators. Subgroups indicate side (i.e., anterior, posterior, medial, and lateral perforators). Thus, the complete name of the perforator is a combination of the level and side (i.e., the medial leg perforator or the anterior thigh perforator).

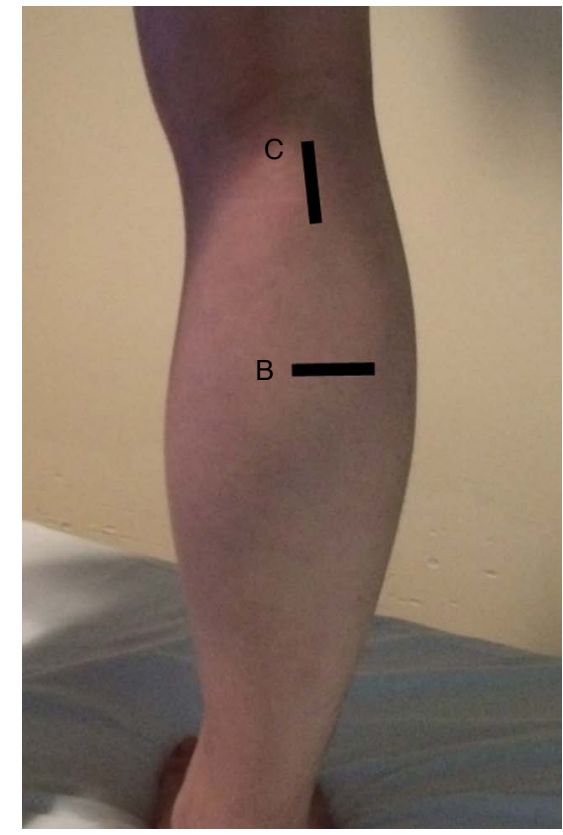

A

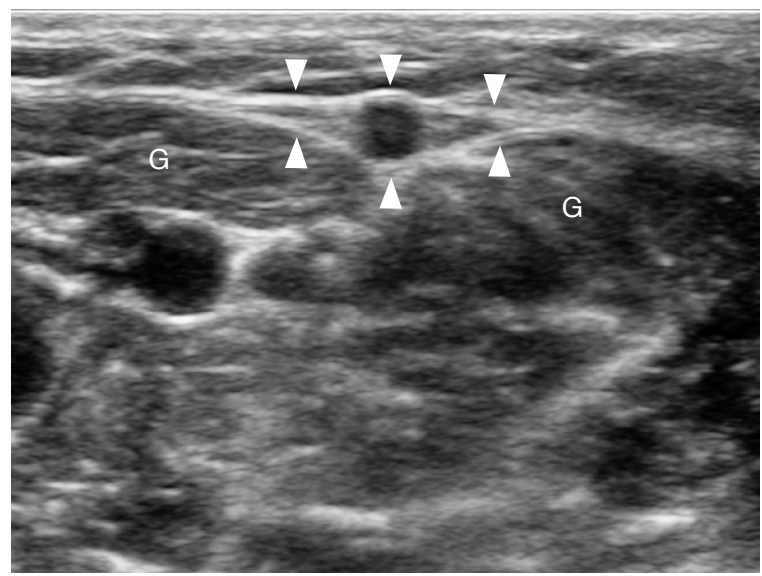

B

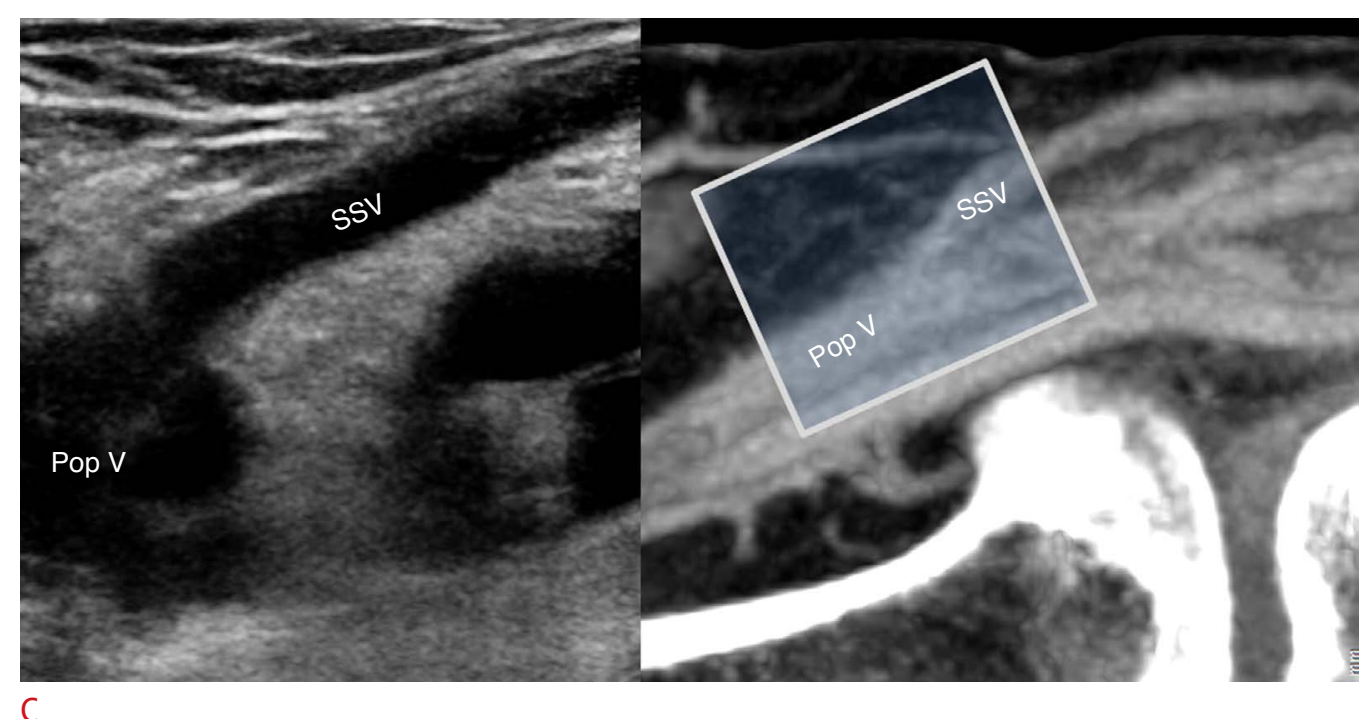

Fig. 8. Ultrasonographic findings of the small saphenous vein (SSV).

A. Patient position and schematic representation of the transducer locations are shown here. B. In the transverse view of the posterior calf, the SSV is seen in the middle of the gastrocnemius (G) belly in the fascial trunk (arrowheads). C. Longitudinal ultrasound view and the corresponding sonic window in computed tomography venography based on transducer location are demonstrated. The SSV joins the popliteal vein (Pop V) through the saphenopopliteal junction. However, many variations are noted in this region, and the saphenopopliteal junction can be absent or hypoplastic. 
More detailed subgroups are present for medial thigh and medial leg perforators. The medial thigh perforators are further classified as the perforating vein of the femoral canal and inguinal perforating vein, and the medial leg perforators are subdivided into the paratibial and posterior tibial perforating veins (Fig. 9). This is convenient becausez it eliminates eponyms such as Hunterian, Dodd, Boyd or Cockett, all

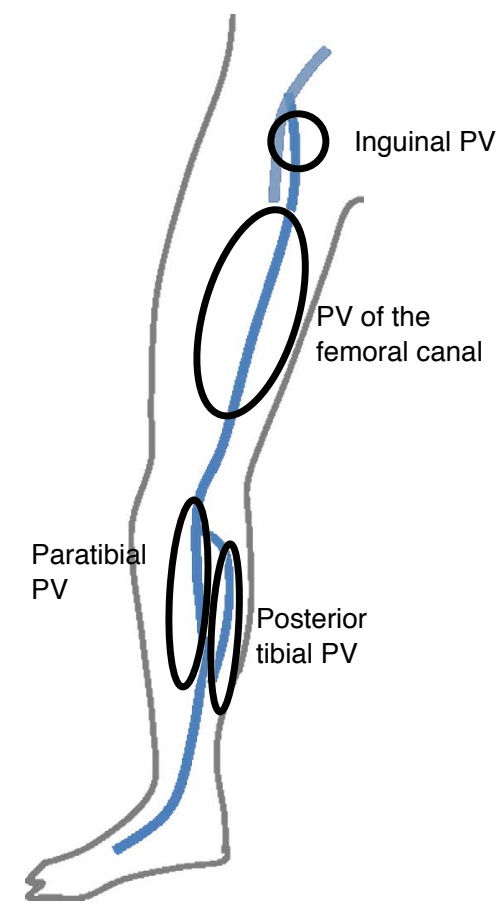

Fig. 9. Schematic images of the perforating veins (PVs) along the medial aspect of the lower extremity. The PV of the medial leg includes the paratibial PV and the posterior tibial PV. The medial thigh perforator includes the PV of the femoral canal and the inguinal PV. of which have been used commonly, but are confusing.

\section{Approach}

Normally, the perforating veins are not clearly visible on US. However, when there is a flow disturbance in the deep or superficial veins or in the case of perforator insufficiency, the perforating veins can be dilated and are readily visible as a penetrating structure through the muscular fascia (Fig. 10A). In the case of varicose veins caused by perforating vein insufficiency, it is important to localize the refluxing point in order to correct it.

This review describes several clinically relevant perforators around the GSV and their connections. The medial thigh and leg perforators are clinically the most important. The medial leg perforator includes the posterior tibial perforator and the paratibial perforator. The posterior tibial perforator, previously known as the Cockett perforator, connects the posterior accessory GSV with the posterior tibial vein in the distal calf. The paratibial perforator connects the GSV or its tributaries with the posterior tibial vein. The proximal paratibial perforator was previously called the Boyd perforator. In the thigh, the perforating vein of the femoral canal, previously called the Hunterian (proximal thigh) or Dodd (distal thigh) perforator, connects the GSV with the femoral vein or the proximal popliteal vein. Insufficiency of these perforators may cause medial thigh and calf varicosities, even in the absence of saphenofemoral reflux (Fig. 10B).

\section{Conclusion}

The venous system of the lower extremities is divided into three groups: deep, superficial, and perforating veins. In US examination

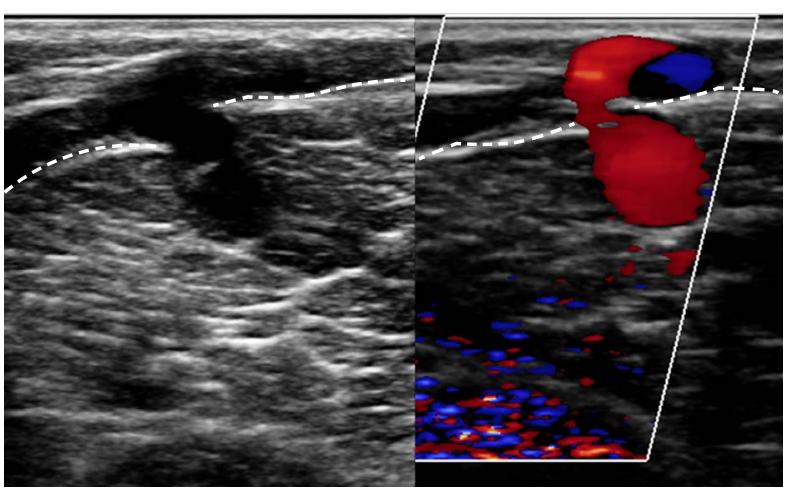

A

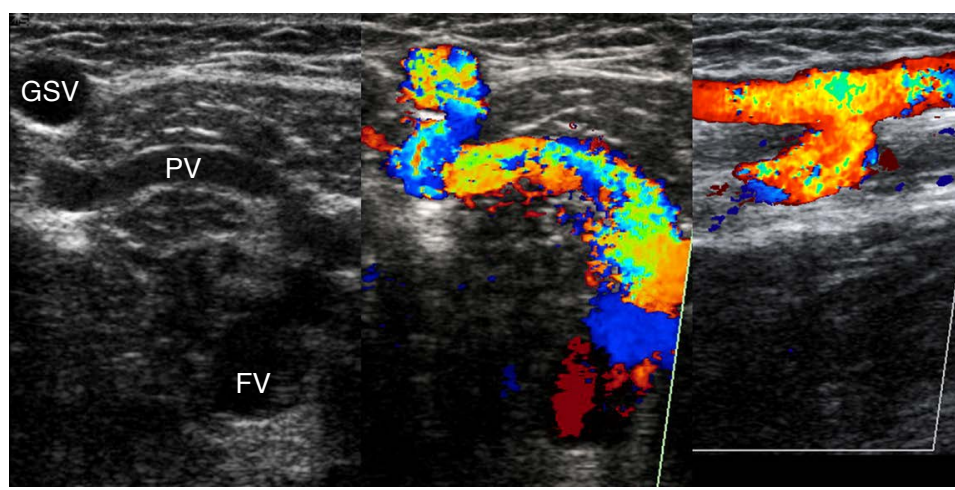

B

Fig. 10. Ultrasonographic findings of perforating veins (PVs).

A. A dilated paratibial perforating vein in the calf is shown here, which penetrates the muscular fascia (dotted lines). B. A dilated PV of the femoral canal is shown here, which connects the great saphenous vein (GSV) and the femoral vein (FV). Venous reflux from the deep to the superficial vein is seen on transverse and longitudinal views of Doppler ultrasonography. 
of the lower extremity veins, knowledge of the venous anatomy as well as appropriate patient positioning and transducer placement are important for optimal imaging and accurate diagnosis.

ORCID: Kyung-Sik Ahn: http://orcid.org/0000-0001-9354-5699

\section{Conflict of Interest}

No potential conflict of interest relevant to this article was reported.

\section{Supplementary Material}

Supplementary Fig. 1. Transverse ultrasonography of an 88-yearold man with a pulmonary embolism. The patient shows duplication of the popliteal vein and thrombosis (arrowheads) in one of the popliteal lumens. On the compression view, the nonthrombosed lumen shows complete obliteration (arrows), while the thrombosed lumen is not fully compressed (open arrows) (https://doi. org/10.14366/usg.17001).

Supplementary Fig. 2. Competent (A) and incompetent (B) saphenofemoral junction. A. After initiation of the Valsalva maneuver, there was no retrograde flow in the proximal great saphenous vein. B. In contrast, retrograde flow that persisted over 3 seconds was observed during the Valsalva maneuver, which suggests abnormal function of the terminal valve (https://doi.org/10.14366/usg.17001).

\section{References}

1. Guideline developed in collaboration with the American College of Radiology; Society of Pediatric Radiology; Society of Radiologists in Ultrasound. AIUM practice guideline for the performance of peripheral venous ultrasound examinations. J Ultrasound Med 2015;34:1-9.

2. Coleridge-Smith P, Labropoulos N, Partsch H, Myers K, Nicolaides A, Cavezzi A. Duplex ultrasound investigation of the veins in chronic venous disease of the lower limbs: UIP consensus document. Part I. Basic principles. Eur J Vasc Endovasc Surg 2006;31:83-92.

3. AbuRahma AF, Bandyk D. Noninvasive vascular diagnosis: a practical guide to therapy. 3rd ed. New York: Springer, 2012.

4. Meissner MH. Lower extremity venous anatomy. Semin Intervent Radiol 2005;22:147-156.

5. Oguzkurt L. Ultrasonographic anatomy of the lower extremity superficial veins. Diagn Interv Radiol 2012;18:423-430.

6. Cina A, Pedicelli A, Di Stasi C, Porcelli A, Fiorentino A, Cina G, et al. Color-Doppler sonography in chronic venous insufficiency: what the radiologist should know. Curr Probl Diagn Radiol 2005;34:51-62.

7. Sigel B, Machi J, Beitler JC, Justin JR, Coelho JC. Variable ultrasound echogenicity in flowing blood. Science 1982;218:1321-1323.

8. Rastegar R, Harnick DJ, Weidemann P, Fuster V, Coller B, Badimon $\mathrm{JJ}$, et al. Spontaneous echo contrast videodensity is flow-related and is dependent on the relative concentrations of fibrinogen and red blood cells. J Am Coll Cardiol 2003;41:603-610.

9. Bundens WP, Bergan JJ, Halasz NA, Murray J, Drehobl M. The superficial femoral vein: a potentially lethal misnomer. JAMA 1995;274:1296-1298.

10. Caggiati $A$, Bergan JJ, Gloviczki $P$, Jantet $G$, Wendell-Smith $C P$, Partsch $\mathrm{H}$, et al. Nomenclature of the veins of the lower limbs: an international interdisciplinary consensus statement. J Vasc Surg 2002;36:416-422.

11. Caggiati A, Bergan JJ, Gloviczki P, Eklof B, Allegra C, Partsch H, et al. Nomenclature of the veins of the lower limb: extensions, refinements, and clinical application. J Vasc Surg 2005;41:719-724.

12. Lin EP, Bhatt S, Rubens D, Dogra VS. The importance of monophasic Doppler waveforms in the common femoral vein: a retrospective study. J Ultrasound Med 2007;26:885-891.

13. Quinlan DJ, Alikhan R, Gishen P, Sidhu PS. Variations in lower limb venous anatomy: implications for US diagnosis of deep vein thrombosis. Radiology 2003;228:443-448.

14. Cavezzi A, Labropoulos N, Partsch H, Ricci S, Caggiati A, Myers $\mathrm{K}$, et al. Duplex ultrasound investigation of the veins in chronic venous disease of the lower limbs: UIP consensus document. Part II. Anatomy. Eur J Vasc Endovasc Surg 2006;31:288-299.

15. Min RJ, Khilnani NM, Golia P. Duplex ultrasound evaluation of lower extremity venous insufficiency. J Vasc Interv Radiol 2003;14:12331241.

16. Khilnani NM, Min RJ. Imaging of venous insufficiency. Semin Intervent Radiol 2005;22:178-184. 